\title{
Light Element Analysis in Extraterrestrial Materials using Secondary Ion Mass Spectrometry
}

Maitrayee Bose

\section{Arizona State University, Tempe, Arizona, United States}

I will present my research on the measurement of light elements (e.g., hydrogen, lithium, boron, carbon \& nitrogen) in extraterrestrial materials and terrestrial analogs using nano-scale secondary ion mass spectrometry (NanoSIMS 50L) at Arizona State University (ASU). The NanoSIMS is part of a NSFfunded National Facility led by Dr. Richard L. Hervig, and also includes an IMS-6f.

The NanoSIMS instrument allows precise isotopic and elemental measurements of sub-micron areas, grains or inclusions from meteorites, micrometeorites, interplanetary dust particles, mineral sections, and synthesized products. It will be instrumental in the characterization of samples to be brought back by ongoing missions, Hayabusa2 and OSIRIS-Rex, which is of particular interest to me.

In my talk, I will discuss our instrument capabilities, including what is required to perform spot analysis and map light elements, even at low concentrations. I will focus on two specific setups that has been developed at ASU to measure hydrogen and lithium:

(1) With the goal to understanding the water contents and $\mathrm{H}$ isotopic compositions of planetary materials, we have successfully measured anhydrous minerals (olivine and pyroxene), as well as hydrous (apatite) minerals within tiny particles collected from asteroid Itokawa and meteorites from 4 Vesta $[1,2]$. We tested new procedures to do samples preparation that is crucial for these complex hydrogen analyses. It has resulted in routine measurements with low hydrogen backgrounds $(<20 \mathrm{ppm})$ with small beam sizes $(<1-2 \mu \mathrm{m})$.

(2) We have developed the methodology to measure Li concentration profiles, and Li isotopes in natural zircons, coal and shale. Zircons are rich in rare earth elements and trace elements, including Li. The diffusion of $\mathrm{Li}$ in zircon crystals allow us to determine magma storage conditions and constrain the mechanisms by which Li can diffuse within zircons [3, 4]. 100s of ppb of Li can be measured via line scans by scanning the primary beam movement along the regions of interest [4], although spot analysis via stage movements were required for high-precision $(<3 \%$ ) lithium isotopes [3]. More recently, we have used multi-isotope maps, including that of $\mathrm{Li}$ to trace gas production in shale and localize $\mathrm{Li}$ in coal [5].

The need for appropriate matrix-matched standards for both $\mathrm{H}$ and $\mathrm{Li}$ isotopes has led to our mission to synthesize ion implant standards, with the intention to sharing material with the community.

References

[1] "New clues to ancient water on Itokawa" (2019) Ziliang J. and Bose M. Science Advances, 5, eaav8106.

[2] "A deuterium-poor water reservoir in the asteroid 4 Vesta and the inner Solar System" (2020) Stephant A., Wadhwa M., Hervig R., Bose M., Zhao X., Barrett T. J., Anand M., Franchi I. A., In press (https://doi.org/10.1016/j.gca.2021.01.004), Geochimica et Cosmochimica Acta.

[3] "Multi-mode Li diffusion in natural zircons" (2017) Tang M., Rudnick R. L., McDonough W. F., Bose M., Goreva Y. Earth and Planetary Science Letters, 474, 110-119.

[4] "Rapid cooling and cold storage in the silicic magma reservoir recorded in individual crystals" (2017) Rubin A. E., Cooper K. M., Till C. B., Kent A. J. R., Costa F., Bose M., Gravley D., Deering C., and Cole J. Science 356, 1154-1156. 
[5] "Lithium isotope compositions of U.S. coals and source rocks: Potential tracer of hydrocarbons" (2020) Teichert Z, Bose M., Williams L. Chemical Geology, 549, 119694-119705. 